\title{
A Novel Approach for SLM based PAPR Reduction using Genetic Algorithm
}

\author{
Mukesh Tiwari \\ Assoc. Professor, ECE \\ Shri Satya Sai Institute of \\ Science \& Technology \\ Sehore (M.P)
}

\author{
Jaikaran Singh \\ Assoc. Professor, ECE \\ Shri Satya Sai Institute of \\ Science \& Technology \\ Sehore (M.P)-India
}

\author{
Lokendra Vishwkarma \\ M.Tech Scholar (DigitalComm.) \\ Shri Satya Sai Institute of \\ Science \& Technology \\ Sehore (M.P)-India
}

\begin{abstract}
An SLM (Selected Mapping) is an effective algorithm to reduce PAPR (Peak to Average Power Ratio) in OFDM (orthogonal frequency division multiplexing) signal without distortion. However the SLM scheme needs to dispose U paths of IFFT (Inverse Fourier Transform), which increases the computational burden and reduces the signal transmission rate. This paper offers an improved algorithm that based on Genetic Algorithm. The proposed algorithm executes the selection before the IFFT module and only selects one sequence with the highest randomness to be transmitted. Because only one path of signal is transmitted in IFFT modules, the proposed algorithm has lower complexity compares with SLM. Moreover, it has advantages in enhancing the signal transmission rate and decreasing the PAPR of OFDM signals.
\end{abstract}

\section{General Terms}

SUBCARRIERS. SELECTIVE MAPPING

\section{Keywords}

SLM, PAPR, OFDM, IFFT, Genetic Algorithm.

\section{INTRODUCTION}

In recent years orthogonal frequency division multiplexing (OFDM) has gained a lot of involvement in diverse digital communication applications. It is a new ensuring transmission scheme for broadband communications over a wireless channel. In OFDM data is transmitted simultaneously through multiple frequency bands [1]. It offers many advantages over single frequency transmission such as high spectral efficiency, robustness to channel fading, immunity to impulse interference, and the capability to handle frequency-selective fading without resorting to complex channel equalization schemes. OFDM also uses small guard interval, and its ability to combat the ISI problem. So, simple channel equalization is needed instead of complex adaptive channel equalization.

In the conventional serial data transmission system, the information symbols are transmitted sequentially where each symbol occupies the entire available spectrum bandwidth. But in an OFDM system, the information is converted to $\mathrm{N}$ parallel sub-channels and sent at lower rates using frequency division multiplexing. The subcarrier frequency spacing is selected carefully such that each subcarrier is located on the other subcarriers zero crossing points [2]. This implies that there is overlapping among the subcarriers but will not interfere with each other, if they are sampled at the sub carrier frequencies. This means that all subcarriers are orthogonal.

Despite the fact that OFDM has a number of advantages, one of the major drawbacks of OFDM signal is its large envelope fluctuation, likely resulting in large peak-to-average power ratio (PAPR) [3]. When the OFDM signals with high PAPR are transmitted through a nonlinear device, such as a highpower amplifier (HPA) or a digital-to-analog converter (DAC), a high peak signal generates out-of-band energy and in-band distortion. These degradations would seriously affect the performance of OFDM systems. The PAPR reduction scheme changes the formation of the OFDM signals with high PAPR before multicarrier modulation, e.g. coding, and selective mapping (SLM). The researches [4]-[6] reduce the computational complexity for the SLM scheme. In SLM we can get better PAPR by modifying the OFDM without distortion. This paper offers an improved algorithm which is based on SLM, is named Genetic Algorithm based SLM (GASLM) and this executes the selection before the IFFT module.

\section{PAPR of OFDM}

The PAPR of OFDM is defined as the ratio between the maximum power and the average power, The PAPR of the OFDM signal $\mathrm{X}(\mathrm{t})$ is defined as

$\mathrm{PAPR}=\frac{\mathrm{P}_{\text {peak }}}{\mathrm{P}_{\text {average }}}=\frac{\max \left[\left|\mathrm{x}_{\mathrm{n}}\right|^{2}\right]}{\mathrm{E}\left[\left|\mathrm{x}_{\mathrm{n}}\right|^{2}\right]}$

Where $x_{n}=$ An OFDM signal after IFFT (Inverse Fast Fourier transform)

E [.] = Expectation operator, it is an average power. The complex baseband OFDM signal for $\mathrm{N}$ subcarriers represented as

$$
\begin{aligned}
& \mathrm{X}(\mathrm{t})=\frac{1}{\sqrt{N}} \sum_{n=0}^{N-1} X_{n} e^{j 2 \pi n \Delta f t},(0 \leq \mathrm{t} \leq \mathrm{NT}) \\
& \text { Selective mapping }(S L M)
\end{aligned}
$$

In selective mapping (SLM) technique [7] the actual transmit signal lowest PAPR is selected from a set of sufficiently different signals which all represents the same information. SLM Techniques are very flexible as they do not impose any restriction on modulation applied in the subcarriers or on their number. Block diagram of SLM Technique is shown in Figure1. 
Let us define data stream after serial to parallel conversion as $\mathrm{X}=\left[\mathrm{X}_{0}, \mathrm{X}_{1} \ldots \ldots \ldots, \mathrm{X}_{\mathrm{N}-1}\right]^{\mathrm{T}}$. Initially each input $\mathrm{X}_{\mathrm{n}}^{(\mathrm{u})}$ can be defined as equation

$$
x_{n}^{(u)}=x_{n} \cdot b_{n}^{(u)}
$$

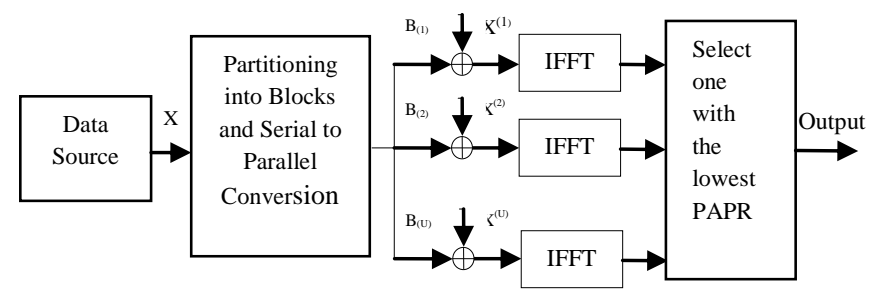

Figure1: Block Diagram of OFDM transmitter with the SLM Technique

$\mathrm{B}^{(\mathrm{u})}$ can be written as $\mathrm{x}_{\mathrm{n}}^{(\mathrm{u})}=\left[\mathrm{x}_{0}^{(\mathrm{u})}, \mathrm{x}_{1}^{(\mathrm{u})}, \ldots \ldots, \mathrm{x}_{\mathrm{N}-2}^{(\mathrm{u})}\right]^{\mathrm{T}}$

Where $\mathrm{n}=0,1,2 \ldots \ldots \mathrm{N}-1$ and $(\mathrm{u}=0,1,2 \ldots \ldots \mathrm{U})$ to make the $\mathrm{U}$ phase rotated OFDM data blocks. All U phase rotated OFDM data blocks represented the same information as the unmodified OFDM data block provided that the phase sequence is known.

After applying the SLM technique, the complex envelope of the transmitted OFDM signal becomes

$\mathrm{x}(\mathrm{t})=\frac{1}{\sqrt{N}} \sum_{n=0}^{N-1} x_{n} e^{j 2 \pi n \Delta f t},(0 \leq \mathrm{t} \leq \mathrm{NT})$

Where $\Delta \mathrm{f}=\frac{1}{\mathrm{NT}}$, and NT is the duration of an OFDM data block. Output data of the lowest PAPR is selected to transmit. PAPR reduction effect will be better as the copy block number $U$ is increased. SLM method effectively reduces PAPR without any signal distortion. But it has higher system complexity and computational burden. This complexity can less by reducing the number of IFFT block.

\section{PROPOSED ALGORITHM}

In order to obtain more effective reduction in PAPR, the SLM scheme would select one sequence after IFFT modules, thus it need N IFFT modules, which makes system highly complicated. According to [8], the more random the sequence is, the smoother the frequency spectrum will become. Thus in order to reduce the complexity of this system, we propose an improved algorithm which is called Selective Mapping with Genetic Algorithm. This scheme selects one sequence with the best randomness before IFFT modules, thus only one IFFT module is necessary, saving the complexity of the system. The principle of GA-SLM can be expressed as followed:

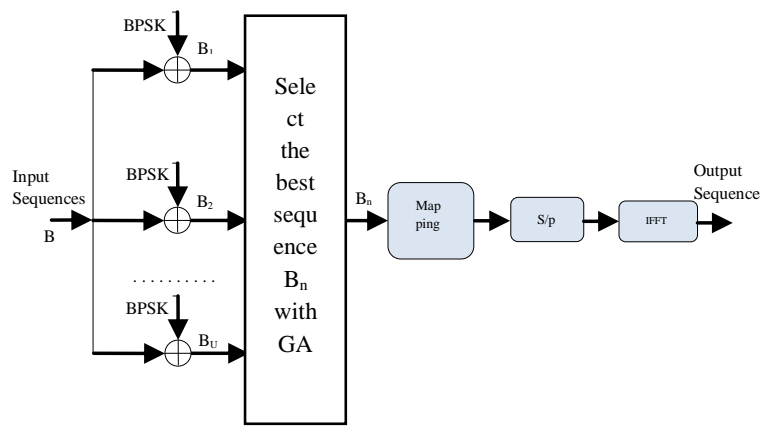

Figure 2: Block diagram of GA-SLM principle

The specific process of the GA-SLM scheme is represented as followed. Suppose the input random sequence B is:

$$
\mathrm{B}=\left(\mathrm{x}_{1}, \mathrm{x}_{2}, \mathrm{x}_{3}, \ldots \ldots, \mathrm{x}_{\mathrm{N}}\right)
$$

Where the number of subcarriers is $N, x_{n}(1 \leq n \leq N)$, which is either 0 or 1 . The BPSK is adopted to modulate the input signal sequences as:

$\mathrm{B}=\left(\mathrm{x}_{\mathrm{n}, 1}, \mathrm{x}_{\mathrm{n}, 2}, \mathrm{x}_{\mathrm{n}, 3}, \ldots \ldots ., \mathrm{x}_{\mathrm{n}, \mathrm{N}}\right) \quad(1 \leq \mathrm{n} \leq \mathrm{U})$

$B_{n}$ is composed of 1 and -1 , GA-SLM selects one sequence which has the best randomness as the output. In this scheme the key point is judge the extent in randomness of the sequence:

1. Judging the nature of randomness of $B_{n}$ sequences. The closer the number of 1 and -1 is, the more random the sequence is, supposing:

$$
\mathrm{S}_{\mathrm{n}}=\sum_{i=1}^{N} x_{n, i} \quad(1 \leq \mathrm{n} \leq \mathrm{U})
$$

Where $S_{n}>0$ represents the numbers of 1 that is greater than $1, S_{n}<0$ stands for the opposite scenario. The $S_{n}$ approaches 0 , the randomness of the sequence increases.

2. Judging the numbers of $B_{n}$ sequence's oscillation which is adding the number of sequence's transitions first, and then compare it with half of the sequence's width. Suppose:

$\mathrm{T}_{\mathrm{n}}=\sum_{\mathrm{i}=1}^{\mathrm{N}-1}\left|\frac{\mathrm{x}_{\mathrm{n}, \mathrm{i}+1}-\mathrm{x}_{\mathrm{n}, \mathrm{i}}}{2}\right|-\frac{\mathrm{N}}{2} \quad(1 \leq \mathrm{n} \leq \mathrm{U})$

Then when the $T_{n}$ is smaller, the nature of random of the sequence is better.

3. Removing the sequences that has a smaller period, As the sequences with short period usually have a high PAPR, we need to exclude them, suppose:
$\mathrm{W}_{\mathrm{n}, 1}=\sum_{k=1}^{N-2} x_{n, k}, x_{n, k+2}$
$(1 \leq \mathrm{n} \leq \mathrm{U})$
$\mathrm{W}_{\mathrm{n}, 2}=\sum_{k=1}^{N-3} x_{n, k}, x_{n, k+3}$
$(1 \leq \mathrm{n} \leq \mathrm{U})$ 


\section{Genetic Algorithm}

Genetic algorithm is a part of evolutionary computing, which is a rapidly growing area of artificial intelligence. We can see that, genetic algorithm is inspired by Darwin's theory about evolution. Simply said, solution to a problem solved by genetic algorithm is evolved. In a genetic algorithm, a population of strings (called chromosomes or the genotype of the genome), which encode candidate solutions (called individuals, creatures, or phenotypes) to an optimization problem, is evolved toward better solutions. Traditionally, solutions are represented in binary as strings of $0 \mathrm{~s}$ and $1 \mathrm{~s}$, but other encodings are also possible.

On the basis on this concept we replace the calculation and give all signals to Genetic Algorithm as input so that optimized signal will have minimum randomness which decided on the basis of $\mathrm{S}_{\mathrm{n}}, \mathrm{T}_{\mathrm{n}}, \mathrm{W}_{\mathrm{N}, 1}$ and $\mathrm{W}_{\mathrm{N}, 2}$ parameter thus at the end it will get selected for further processing.

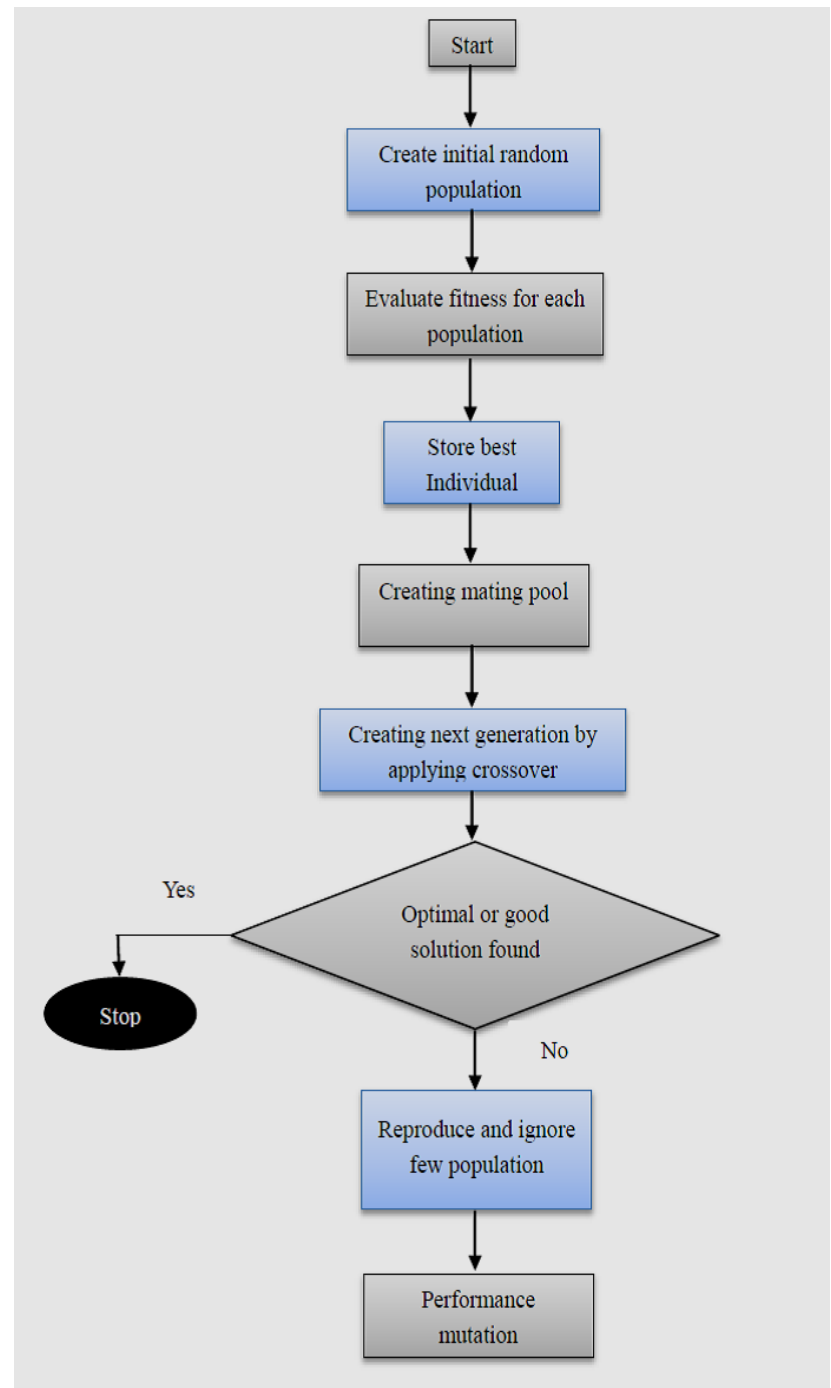

Figure 3: Flow chart of GA

\section{SIMULATION AND RESULTS}

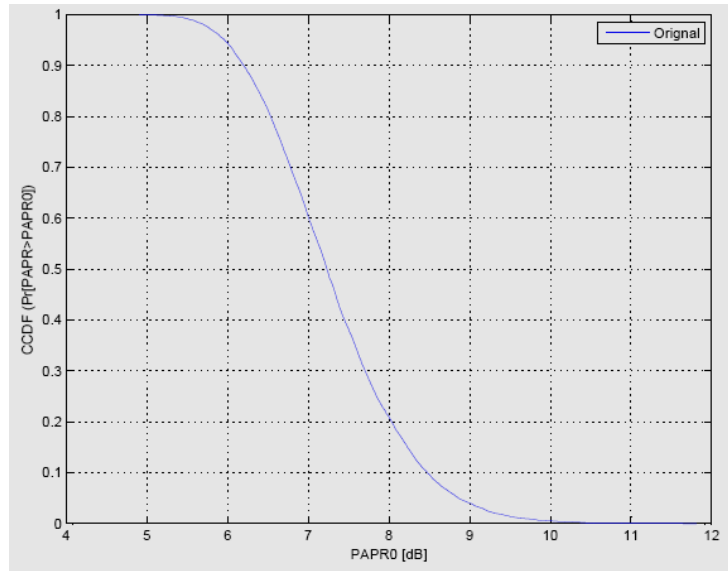

Figure 4: PAPR v/s CCDF graph of original signal

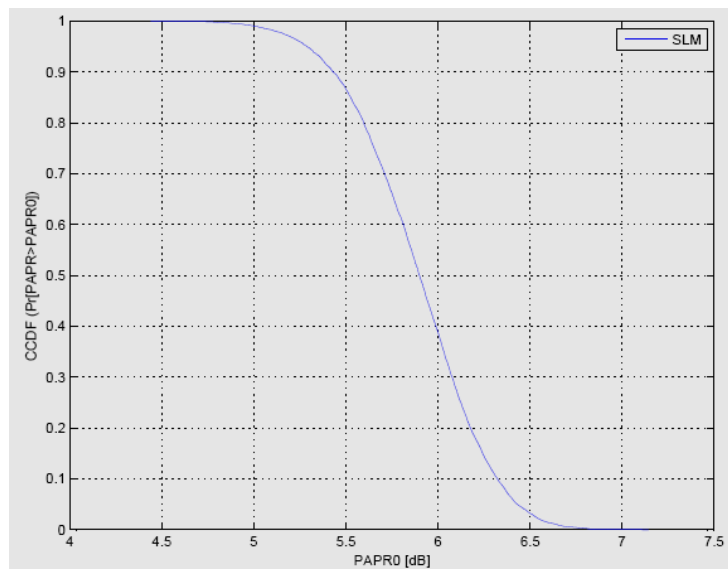

Figure 5: PAPR v/s CCDF graph in selective mapping method

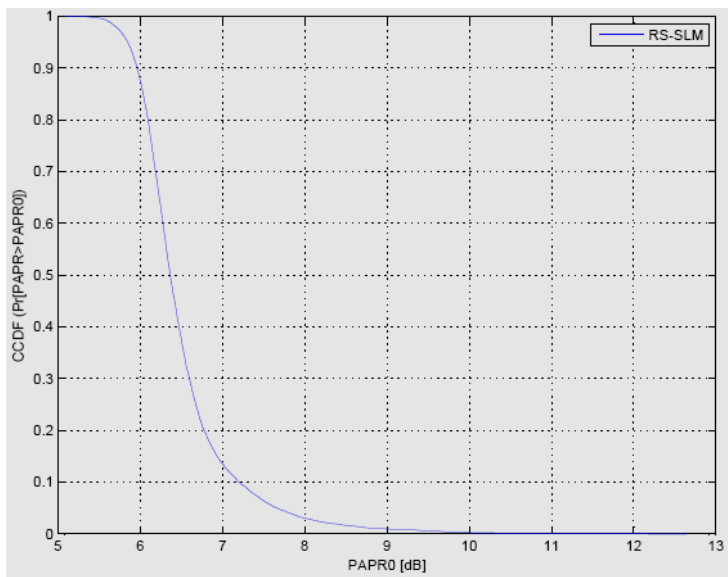

Figure 6: PAPR v/s CCDF graph in RS-SLM 


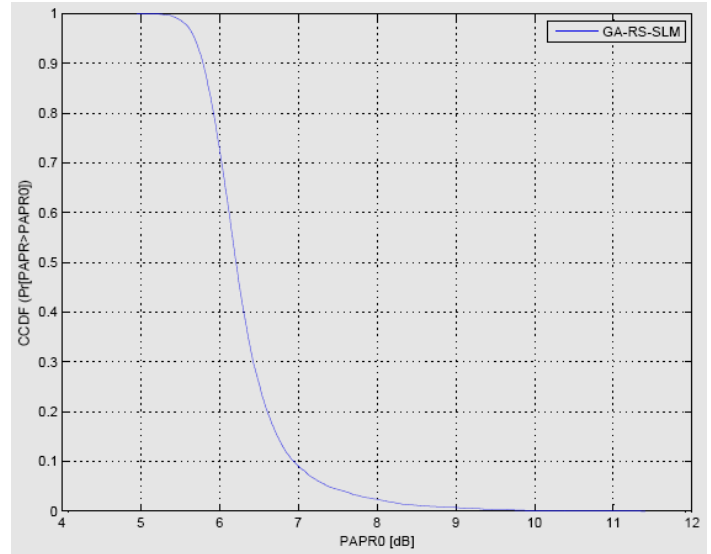

Figure 7: PAPR v/s CCDF graph in GA-RS-SLM

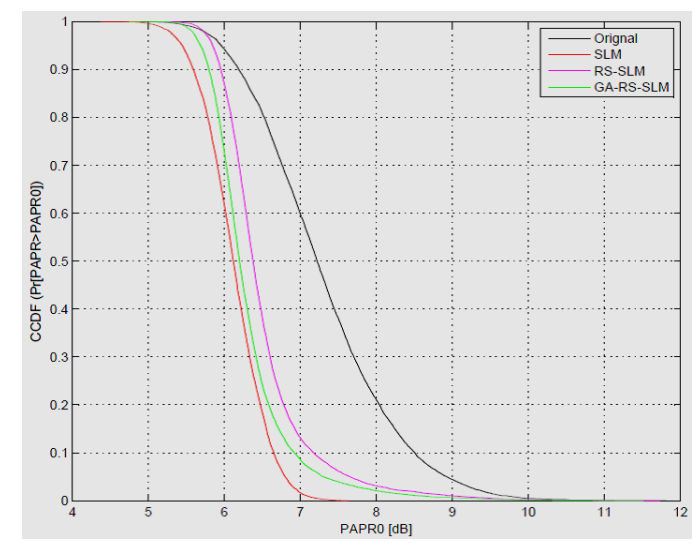

Figure 8: Comparison of different schemes (showing PAPRO v/s CCDF)

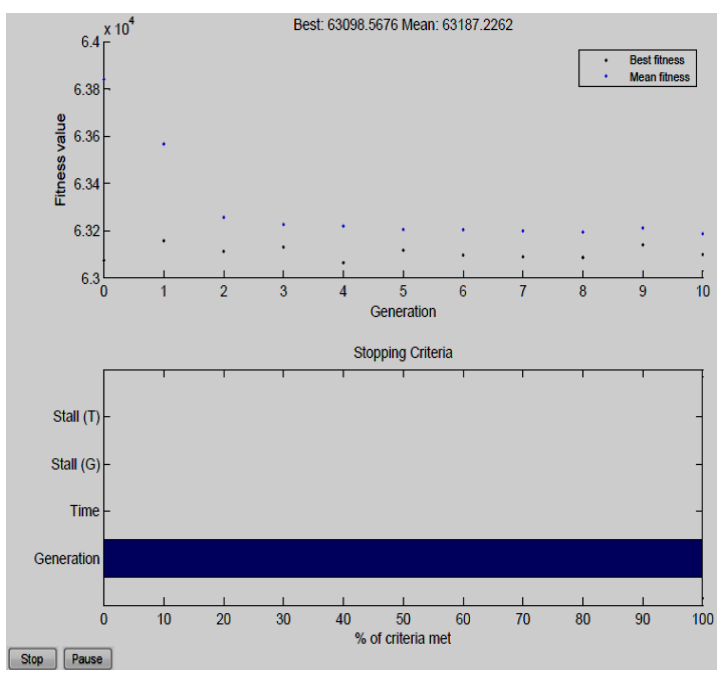

Figure 9: Optimization through Genetic Algorithm

\section{CONCLUSION}

This paper proposes an improved algorithm GA-SLM which is based on SLM. The simulation results predict that the new algorithm is effective in reducing the PAPR of OFDM system. Though its PAPR performance is a little worse than the SLM scheme, this new algorithm can reduce complexity of OFDM system greatly.

\section{REFERENCE}

[1] A. D. S. Jayalath, C. Tellambura and H. Wu, "Reduced complexity PTS and new phase sequences for SLM to reduce PAP of an OFDM signal", Vehicular Technology Conference Proceedings, VTC 2000-Spring Tokyo. 2000 IEEE 51st.

[2] P. Foomooljareon and W. Fernando, "PAPR reduction in OFDM systems," Thammasat International Journal of Science and Technology, vol. 7, no. 3, 2002.

[3] J.Tellado, J.Tellado, "Multicarrier transmission with lowPAR," Ph.D. dissertation, Stanford Univ., Stanford, CA, 1998

[4] C. P. Li, S. H Wang, and C. L. Wang, "Novel lowcomplexity SLM schemes for PAPR reduction in OFDM systems," IEEE Trans. Signal Process., vol. 58, no. 5, pp. 2916-2921, May 2010.

[5] J. H. Wen, S. H Lee, and C. C. Kung, "SLM-based data position permutation method for PAPR reduction in OFDM systems," in Wireless Communication and Mobile Computing. Hoboken, NJ: Wiley Inter-Science, 2008.

[6] S. H. Wang and C. P. Li, "A low-complexity PAPR reduction scheme for SFBC MIMO-OFDM systems," IEEE Signal Process. Lett., vol. 16, no. 11, pp. 941-944, Nov. 2009.

[7] Ms. V. B. Malode, Dr. B. P. Patil, "PAPR Reduction Using Modified Selective Mapping Technique", IJANA, Volume: 02, Issue: 02, Pages: 626-630, 2010.

[8] WANG Wen-bo ZHENG Kan, "The OFDM technique of broadband wireless", Beijing, Post and Telecom press, 2003.

[9] Wang Ding, Xu Lin-Bo, "An Improved SLM Algorithm for Reducing PAPR in OFDM System”, IEEE. 